\title{
Opisthorchis felineus extracellular vesicles increase cell proliferation and migration rates of human H69 cholangiocytes
}

\author{
D.V. Ponomarev*, O. Zaparina, M.Y. Pakharukova, V.A. Mordvinov \\ Laboratory of Molecular Mechanisms of Pathological Processes \\ Institute of Cytology and Genetics SB RAS \\ Novosibirsk, Russia \\ *E-mail: p.dmitr@outlook.com
}

\begin{abstract}
Opisthorchis felineus, is a food-borne liver trematode and the main cause of opisthorchiasis in Russia and Europe. It affects hepatobiliary system of fish-eating mammals, including humans. Opisthorchiasis is associated with chronic inflammation, biliary epithelium proliferation, liver fibrosis, and even might cause cholangiocarcinoma among chronically infected individuals. Secreted proteins and extracellular vesicles of liver flukes might play an important role in the development of pathology. We investigated the response of human H69 cholangiocytes and human hepatoma HepG2 cells to adult liver flukes and to extracellular vesicles released from the flukes. We have demonstrated high mitogenic and cell migration stimulating activity of flukes and EVs vesicles. The activity was specific for cholangiocytes, but not for HepG2 cells. Specific mitogenic effect of liver fluke extracellular vesicles on proliferation and migration of human cholangiocytes in vitro may reflect the mechanisms of development of precancerous biliary intraepithelial neoplasia during opisthorchiasis in vivo.
\end{abstract}

Key words - Opisthorchis felineus, liver flukes, extracellular vesicles, cholangiocytes, cell proliferation

\section{Motivation and aim}

The causative agent of opisthorchiasis Opisthorchis felineus causes chronic inflammation, biliary intraepithelial neoplasia and cholangiofibrosis [1]. Chronic opisthorchiasis may cause cholangiocarcinoma, cancer of the biliary tract. The molecular mechanisms of pathology are not studied. One of the potential key events in host-parasite interaction might be fluke secretory proteins, released from the fluke.

The aim was to investigate the effect of adult worms and their extracellular vesicles on cell proliferation and migration rates of human $\mathrm{H} 69$ cholangiocytes.

\section{Matherial and methods}

Adult Opisthorchis felineus liver flukes were extracted from laboratory hamsters previously infected with the metacercariae. Adult flukes were cultured in the media for 7 days. EVs were extracted from the cultivation media and purified, using ultrafiltration approach. Human SV-40 immortalized cholangiocytes (H69) [2] and human hepatoma cells (HepG2) were used to assess the effects of EVs and adult flukes on cell proliferation and cell migration.

To examine the effect of adult flukes on cell proliferation, 16 flukes were co-cultured with either H69 or HepG2 cells using a non-contact approach in 12-well Transwell plates with a 1 or $8 \mu \mathrm{m}$ pore size chambers. The numbers of cells was calculated at days 4, 9 and 14. Cell migration analysis was performed after 14 days of co-cultivation with adult flukes using traditional scratch test.

\section{Results}

In contrast to HepG2 cells, proliferation of human cholangiocytes H69 was 4-17 times increased after cocultivation with the liver flukes. The rate of H69 cell migration, (not HepG2) was also elevated (2 times) after the liver flukes and was accompanied by cell morphological changes.

The effect of fluke extracellular vesicles on proliferation and rate of cell migration was similar to the effect of adult flukes. Unlike H69 cells, the influence on HepG2 cells was not significant. Specific mitogenic effect of liver fluke extracellular vesicles on proliferation and migration of human cholangiocytes in vitro may reflect the mechanisms of development of precancerous biliary intraepithelial neoplasia during opisthorchiasis in vivo.

\section{ACKNOWLEDGMENT}

The study was financially supported by the Russian Science Foundation No. 18-15-00098.

\section{REFERENCES}

[1] Mariya Y. Pakharukova and Viatcheslav A. Mordvinov, "The liver fluke Opisthorchis felineus: biology, epidemiology and carcinogenic potential," Transactions of The Royal Society of Tropical Medicine and Hygiene, Volume 110, Issue 1, p. 28-36, 06 January 2016., in press

[2] S. A. Grubman, R. D. Perrone, D. W. Lee, S. L. Murray, L. C. Rogers, L. I. Wolkoff et al, "Regulation of intracellular $\mathrm{pH}$ by immortalized human intrahepatic biliary epithelial cell lines," American Physiological Society, pp. G1060-G1070, 1 June 1994., in press 